\title{
The Optimal Pace of Product Updates
}

November 18, 2004

\author{
Cheryl T. Druehl
}

R. H. Smith School of Business

The University of Maryland

College Park, MD 20742

(301) 405-9677

cdruehl@umd.edu

Glen M. Schmidt

The McDonough School of Business

Georgetown University

Washington, DC 20057

(202) 687-4486

schmidtg@msb.edu

Gilvan C. Souza

R. H. Smith School of Business

The University of Maryland

College Park, MD 20742

(301) 405-0628

gsouza@umd.edu 


\title{
The Optimal Pace of Product Updates
}

\begin{abstract}
Some firms (such as Intel and Medtronics) use a time-pacing strategy for new product development, introducing new generations at regular intervals. If the firm adopts a fast pace (introducing frequently) then it prematurely cannibalizes its old generation and incurs high development costs, while if it waits too long, it fails to capitalize on customer willingness-topay for more advanced technology. We develop a model to gain insight into which factors drive the pace. We consider the degree to which a new generation stimulates market growth, the rate at which it diffuses (its coefficients of innovation and imitation), the rate of decline in its margin over time, and the cost of new product development. The optimization problem is non-concave; however we are able to solve it numerically for a wide range of parameters because there is a finite number of possible solutions for each case. Somewhat intuitively, we find that a faster pace is associated with a higher market growth rate and faster margin decay. Not so intuitively, we find that relatively minor differences in the new product development cost function can significantly impact the optimal pace. Regarding the Bass coefficients of innovation and imitation, we find that a higher sum of these coefficients leads to a faster pace but with diminishing effects, and that for relatively higher sums the coefficients are effectively substitutes.
\end{abstract}

Keywords: new product introduction, diffusion, time-pacing, clockspeed 


\section{Introduction}

Consider a firm such as Intel that periodically updates its product line with a new generation of product. For example, since the introduction of the early 4004 microprocessor, Intel has typically introduced a new generation every three to four years as shown in Figure 1. Each new technologically-advanced generation revitalizes the product line, initially commanding a relatively higher price. But then the price begins to decay as the new generation diffuses through the market, displacing the previous generation. As it ages, the new generation itself eventually becomes ripe for replacement.

Figure 1: Intel's new product development launch history (Intel 2004)

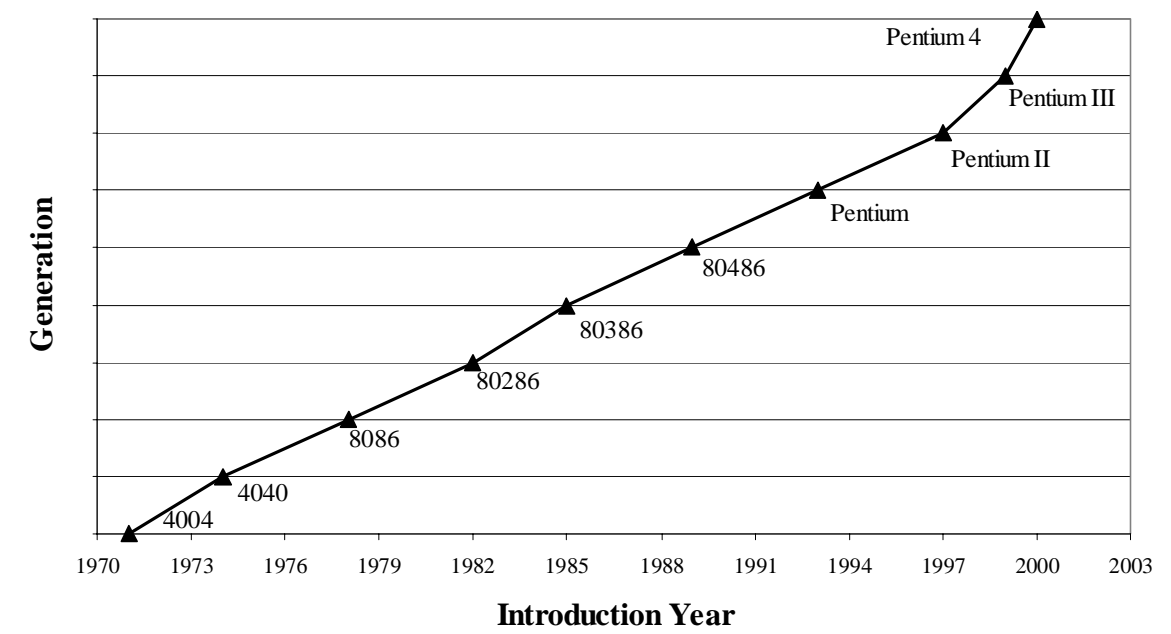

Many electronic products are similarly updated at relatively regular intervals, including items such as computer components, printers and other peripherals, digital cameras, and cell phones. From a customer's perspective, these products seem to improve at a somewhat regular pace, as new generations of products are repeatedly introduced. Eisenhardt and Brown (1998) refer to this type of product development strategy as time pacing. A notion similar to that of the pace of new product introduction is that of industry clockspeed as discussed by Fine (1998) and by Mendelson and Pillai (1999): a fast pace would contribute to a fast clockspeed. 
The pace at which a company introduces new product generations is an important but complex decision for the firm. For example, say Intel has just introduced a new generation of microprocessor. It then needs to decide whether to introduce the next generation microprocessor next year, in two years, or at some point further down the road. If it goes for an early introduction, it may incur high costs (in product development, for example) and it may prematurely cannibalize its previous generation. If it waits too long, it may fail to capitalize on customer willingness-to-pay for more advanced technology, in addition to the possibility that competitors may (further) infiltrate the market. Additionally, as discussed by Kornish (2001) and Krishnan and Ramachandran (2004), the pacing decision may impact whether customers who would otherwise immediately buy might instead choose to wait for the upgraded product. How frequently should it introduce a new generation?

We develop a model to analyze some of the firm's trade-offs. We focus on the interaction of seven parameters: the shape and scale of the product development (PD) cost curve, the Bass diffusion coefficients of innovation and imitation (reflecting the impact of cannibalization and sales growth within a generation's life), the rate of growth in potential market size, the rate of decline in profit margin after a new product's introduction, and the discount rate. We examine each of the parameters at three levels in a full-factorial experiment. For each of the 2,187 runs we find the profit-maximizing pace through an exhaustive numerical search-although the optimization problem is non-concave, it has a finite number of possible solutions.

With regard to the above factors, we make the following assumptions. First, we assume the PD cost is convex ("crashing" is costly while extensively long development times also lead to inefficiencies in PD—see Bayus 1997 and Graves 1989). Second, to model the diffusion process we follow the multi-generation model of Norton and Bass (1987), hereafter abbreviated 
N/B. Third, similar to N/B we assume the overall market size grows incrementally over time (or remains constant). And fourth, we assume the profit margin for a given generation of product declines exponentially over time_-see Bayus (1997), Krishnan et al. (1999), Smith and Reinertsen (1991) and Carrillo (2004).

The rates of growth in market size and of decay in profit margin are effectively surrogates for several of the factors mentioned earlier, such as the rate of change in customer willingness-to-pay and the competitive nature of the market. For example, the price of each subsequent upgrade of an Intel microprocessor decays over time as shown in Figure 2. To formally model the margin decay one would also need to consider the pricing strategy and the cost trajectory, which may also be downward due to the learning curve. However, for simplicity, we directly assume an exponential decay in margin.

Figure 2: Price decay after introduction of an Intel microprocessor (Intel 2004)

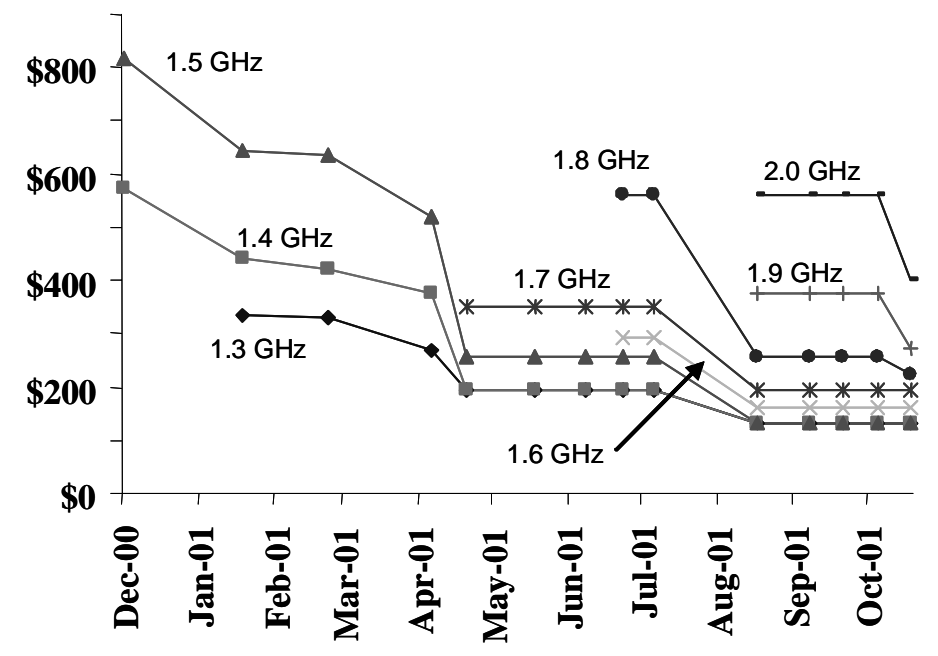

To the best of our knowledge we are the first to consider how the diffusion coefficients of a new generation of product (i.e., the coefficients of innovation and imitation and the market growth rate) impact the time-pacing decision. This is significant in that this diffusion has been empirically verified (N/B 1992), and here we find these coefficients are some of the most important determinants of the pace. In particular, we find that a higher sum of the coefficients of 
innovation and imitation leads to a faster pace but with diminishing effects, and that for relatively higher sums the coefficients are effectively substitutes. In other words, if there is a sufficient base rate of adoption due to external communication (i.e., a sufficiently high coefficient of innovation), then it is only the magnitude of the sum that matters-it doesn't matter whether it is the coefficient of imitation or the coefficient of innovation that contributes to the sum.

Another interesting finding is that seemingly relatively minor differences in the new product development cost curve can significantly impact the optimal pace. Product development cost might typically represent less than $5 \%$ of the total product revenues over a product's life (Ulrich and Eppinger 2004). Yet, we find that subtle differences in the shape of the PD cost curve (which may impact PD cost by only $10 \%$ or so) can result in differences of more than $50 \%$ in the pace of new product introduction. This may help explain why product life cycles apparently have gotten shorter - even a relatively modest improvement in PD cost can significantly alter the pace.

We review additional related literature in $\S 2$. We present the details of our model in $\S 3$, followed by presentation of our numerical studies in $\S 4$. We discuss implications of our results and conclude in $\S 5$.

\section{Literature Review}

Time pacing (Eisenhardt and Brown 1998) has been linked to the idea of clockspeed, Fine's (1998) loosely-defined term denoting the rate at which an industry or firm operates. Souza et al. (2004) define industry clockspeed as the rate of decay in prices, in a model that incorporates competition, demand uncertainty, and inventory. They conclude that time pacing may not be optimal, but generally performs well. Similarly to us, they find that the pace of new 
product introductions increases as industry clockspeed increases. Souza (2004) models a multiperiod game where two profit-maximizing firms compete by introducing new products. He concludes that increased manufacturing learning increases the firm's pace of product introductions. Both Souza et al. (2004) and Souza (2004), however, assume a fixed cost associated with a constant product development time of one period, a single product generation in the market at any time, and do not model diffusion. In contrast, we assume a U-shaped product development cost with time, two generations in the market at any time, and model diffusion.

An extant stream of literature examines the trade-off between product development time, cost and product quality for a single project. Cohen et al. (1996) consider a multi-stage development process and show that the fast introduction of a low-quality product is usually not optimal. Bayus (1997) finds that fast development of low-quality products is optimal only when the market window is short, the competitor is relatively weak, and the development cost curve is relatively steep. In a game-theoretic model, Bayus et al. (1997) find that a profit-maximizing firm with a new technology may choose to be the first to market or wait and enter with a better product, depending on cost and market parameters. Klastorin and Tsai (2004) examine the choice of product design complexity (fixing development time) and price in a duopoly. In contrast to these models, we explicitly model the impact of diffusion shape on the pace of new product introductions in a multi-generation product scenario.

Morgan et al. (2001) extend the study of the cost-quality-time trade-off in product development to multiple generations, but there are key differences with respect to our model. Morgan et al. (2001) assume a constant market size and revenue per unit over time; in contrast our model explores the key trade-offs between diffusion rate, declining margins over time, and a U-shaped product development cost curve. Morgan et al. (2001) find that a short product 
development cycle is strongly associated with a faster industry quality improvement rate, while we somewhat similarly find that higher market growth potential results in a faster pace.

Similarly to our model, Carrillo (2004) investigates the optimal pace of new product introductions. However, she models diffusion using the single-generation generalized Bass model (Bass et al. 1994), does not study different diffusion curve shapes, and assumes a fixed cost for product introduction independent of introduction time. Conversely, we investigate different diffusion curve shapes in a multi-generation scenario, and assume that product introduction cost depends on introduction time, as observed empirically. While Carrillo finds that lower product development costs speed introduction, we find that the shape of the curve is more significant. In addition, Carrillo finds that a faster margin decay results in a slower introduction pace unless the initial revenue goes up to offset the decay; we instead find that faster margin decay always leads to a faster introduction pace, ceteris paribus.

Wilson and Norton (1989) model a situation where a monopolist offers one product, then decides when to introduce a second-generation product in order to maximize profits. A key difference between our models is that we incorporate product development cost into the decision, while they assume the new product is "ready to go" at the beginning of the time horizon. Consequently, they find situations where it is optimal to introduce both products immediately, whereas in our model the firm never introduces the second-generation product immediately due to the extremely high cost of short development times. Furthermore, their planning horizon is shorter than the time it takes for the first product to completely diffuse through the market; in contrast, we assume that the horizon is long enough such that multiple generations of product are generally introduced.

The sequence and timing of new product introductions for two or more products with differing quality levels has been considered as a way to alleviate cannibalism - see Moorthy and 
Png (1992), Chen and Yu (2002), Bhattacharya et al. (2003), and Krishnan and Zhu (2003). Unlike these models, we focus on the optimal time of introduction for the second and subsequent products, assume margin decay over time for each generation, and assume that future products expand the overall market using a multi-generation diffusion model.

In sum, although there is significant research on the timing of new product introduction, our paper is the first to analyze the key trade-offs between diffusion curves, rate of margin decay, and product development cost curve in a multi-generational product scenario.

\section{Model}

Our model finds the optimal pace (i.e., rate) of new product introductions, assuming the firm follows a time-pacing strategy involving constant time intervals between new product generational updates. Time $t$ takes on only integer values, denoting a time period (for example, $t$ $=10$ might denote month 10). We assume the firm can introduce a new generation of product only at the end of a period, with the planning horizon involving $T$ periods. The number of periods between introductions is denoted by $\tau$. We call the inverse of $\tau$ the pace (e.g. if the firm introduces every 24 months, then the pace is 0.5 introductions per year). The optimal pace is denoted by $1 / \tau^{*}$. We assume the firm is a monopolist, or that competition effects can be captured in the product price decline over time. We track our notation in Table 1. 
Table 1: Notation

\begin{tabular}{ll}
\hline Symbol & Description \\
\hline$t$ & Time period, $t=0,1,2, \ldots, T$ \\
$T$ & Time horizon, in periods \\
$\tau$ & Number of periods between introduction of successive generations \\
$1 / \tau^{*}$ & Optimal pace of introductions \\
$t_{i}$ & Time period in which generation $i$ is introduced (at the end of the period); \\
& $t_{i}=(i-1) \tau$ (thus $\left.t_{1}=0\right)$ \\
$r_{i}(t)$ & Profit margin (price - cost) for generation $i$ during period $t ; t \geq t_{i}$ \\
$r_{0}$ & Profit margin in the period a generation is introduced \\
$b$ & Fractional decrease in profit margin per period \\
$D$ & Scale parameter for PD cost curve \\
$d$ & Shape parameter for PD cost curve \\
$\alpha$ & Discount factor \\
$m_{1}$ & Gross potential market for generation 1 \\
$m_{i}$ & Incremental gross potential market for generation $i$ relative to generation \\
& $i-1, i>1$
\end{tabular}

We assume without loss of generality that the first-generation product is introduced at the end of period $t=0$, and denote the introduction of the $i^{\text {th }}$ generation as occurring at the end of period $t_{i}=(i-1) \tau$. We assume the firm only has two generations of product in the market at any one time (e.g. the first generation is dropped when the third is introduced). This assumption is supported, for example, by the observation that when Intel introduced the Pentium IV, shipments of Pentium II dropped to near zero (Dataquest 2003). Thus generation $i$ cannibalizes sales from generation $i-1$, but later has its sales cannibalized by generation $i+1$. Diffusion of the new generation progresses either due to communication or because the new product is better in some 
way. For example, in fashion goods, the latest color and style may be considered by customers to be an improvement over the old version. Similarly, in microprocessors, the new chip has a faster processing speed and more transistors. See Schmidt and Druehl (2004). Regardless of whether it is communications or changes in attributes that cause the diffusion, we apply the multi-generation N/B (1987) model to track the diffusion process (details are given later).

We assume that unit profit margin $r_{i}(t)$ (selling price minus production cost) for each generation $i$ is constant over a given period but decreases exponentially from period to period per the relationship $r_{i}(t)=r_{0} \exp \left(-b\left(t-t_{i}\right)\right)$, where $b$ denotes the rate of profit margin decrease per period. Exponential margin decay is considered a reasonable assumption given manufacturing learning (Yelle 1979) on the cost side, and considering a complex set of factors, such as the pace of technological evolvement, on the price side. Following Bayus (1997) and Smith and Reinertsen (1991), we combine exponential cost and price decay into a single variable for parsimony. Further support, in addition to the available empirical evidence, for our assumption of declining margins over time is found in Bass's (1980) finding that declining prices from the learning curve effect result in faster adoption of new technology and in the finding of Krishnan et al. (1999) that, under certain conditions, a monotonically decreasing price for a product that experiences learning effects is optimal.

We assume that product development (PD) cost for introducing a product in the market is a convex "U-shaped" function of its product development time $\tau$. That is, for introduction time values, $\tau$, that are less than the introduction time where PD cost is minimized, PD cost increases as $\tau$ decreases due to increasing crashing costs to reduce PD time. Conversely, for $\tau$ values greater than the introduction time where PD cost is minimized, PD cost increases as $\tau$ increases due to decreasing know-how, lack of motivation and high setup costs as people move to other 
projects. For a review on the justification for such a shape for the product development cost curve, the reader is referred to Bayus (1997) and Graves (1989).

More specifically, we assume that product development cost is a function of $\tau$ according to the functional form $D[1 /[\exp (d \tau)-1]+d \tau]$, where $D$ is a scaling parameter, and $d$ is a shape parameter that controls both the time of minimum cost, as well as the steepness of the curve (but all values of $d$ yield the same PD cost minimum). Examples are shown in Figure 3. Note that we do not include the PD cost for the first generation.

Figure 3: Product development cost curves

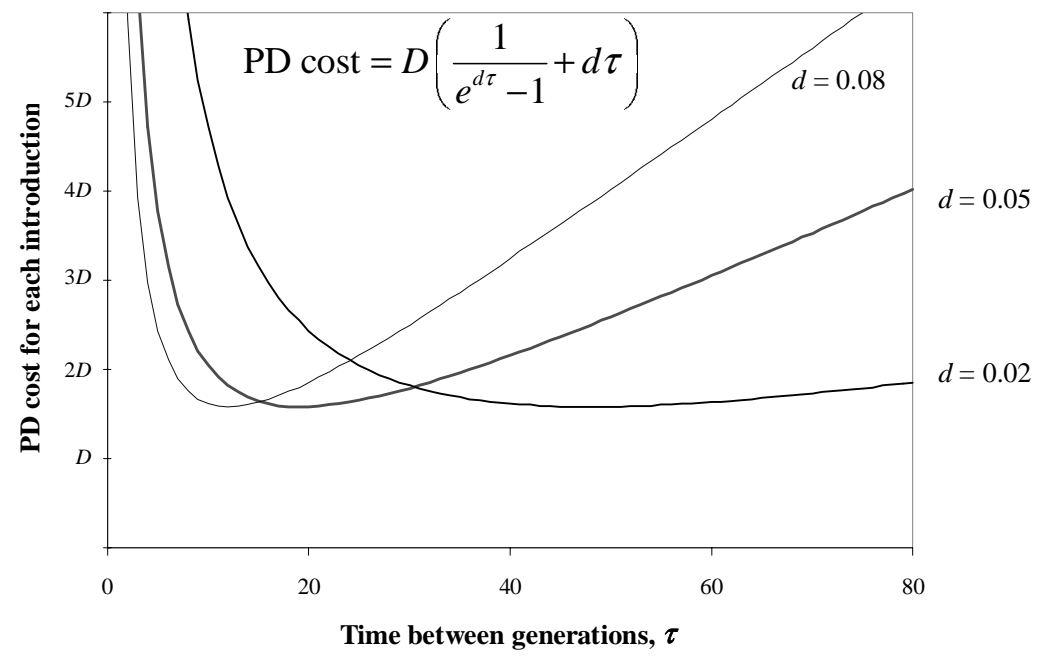

We also experimented with a quadratic form for the PD cost curve, and reached the same conclusions; we prefer the exponential-linear curve above because a single parameter $d$ defines the shape of the curve, and because the PD cost approaches infinity as $\tau$ approaches zero, and as $\tau$ approaches infinity. This formulation eliminates the possibility that two generations are introduced at the same time and assumes that the development of the future generation depends on the previous generation's successful development. For parsimony, we consider that the product development cost is incurred once at the time of each introduction. Finally, a cash flow $F$ in period $t$ is worth $F e^{-\alpha t}$ at period zero (we do not discount within a period). The objective is 
to maximize total discounted profit at period zero, where profit in each period is the sum of the net revenues (sales volume for each generation multiplied by its per-unit profit margin) minus the product development cost (if there is one).

The Bass (1969) model has been applied in numerous settings and extended in many ways. See Mahajan et al. (1990) for a review of these extensions. We use the multi-generation Bass model of Norton and Bass (N/B) (1987, 1992), described briefly as follows.

To find the sales of generation $i$ in period $t$, first define:

$$
F_{i}(t) \equiv \begin{cases}0 & \text { if } \quad t \leq t_{i}, \\ \frac{1-e^{-(p+q)\left(t-t_{i}\right)}}{1+\frac{q}{p} e^{-(p+q)\left(t-t_{i}\right)}} & \text { if } t_{i}<t \leq t_{i+2} \\ 1 & \text { if } t>t_{i+2}\end{cases}
$$

$F_{i}(t)$ can be thought of as the fraction of generation $i$ 's potential market that will purchase generation $i$ in period $t$. Note that if $t<t_{i}$ then generation $i$ has not yet been introduced while if $t=t_{i}$ then it is introduced only at the end of the period so in either case its purchase fraction is zero for that period, while if $t>t_{i+2}$ then its purchase fraction is one, but below we show that in this case generation $i$ 's potential market is zero because the next generation has cannibalized all its potential.

There are two factors contributing to generation $i$ 's potential market size. The first factor, denoted by $Y_{i}(t)$, accounts for the notion that generation $i$ cannibalizes some (or all) of older generation $(i-1)$ 's potential market. For $i>1$ define:

$$
Y_{i}(t) \equiv Y_{i-1}(t) F_{i-1}(t)+m_{i}
$$

where the original potential market is $m_{1}$ (for $i=1$ we define $Y_{1}(t)=m_{1}$ ) and the incremental potential market each period thereafter is denoted $m_{i}, i>1$. In other words, $Y_{i}(t)$ represents all potential purchases of the previous generation, $Y_{i-1}(t) F_{i-1}(t)$, plus some incremental growth $m_{i}$. We call $Y_{i}(t)$ the gross potential market for generation $i$ in period $t$. 
The other factor contributing to generation $i$ 's potential market size is denoted by $Z_{i}(t)$, and it accounts for the fact that generation $i$ may itself be partially (or fully) cannibalized by the newer generation $i+1$. Note that by (1) and (2) a newer generation $i+1$ will cannibalize the fraction $F_{i+1}(t)$ of generation $i$ 's gross potential market. This leaves un-cannibalized for generation $i$ the fraction:

$$
Z_{i}(t)=1-F_{i+1}(t)
$$

The product $Y_{i}(t) Z_{i}(t)$ can be thought of as the net potential market. (Note that as claimed earlier, this is zero for $t>t_{i+2}$. ) The actual market or sales for generation $i$ during time period $t$ is thus:

$$
S_{i}(t)=F_{i}(t) Y_{i}(t) Z_{i}(t)
$$

We effectively assume that sales progress at the same rate over the duration of each period, with the rate given by (4). Assuming the firm is a monopolist, it will sell $S_{i}(t)$ in period $t$. If there is competition, a possible interpretation of our assumptions is that the firm's market share is constant over the horizon (the total market is scaled up from $S_{i}(t)$ ).

To simulate the way in which a new generation of product often expands the market, we consider that the potential for a new generation to increase the market size grows at a rate of $g \geq$ 0 per period, where $g=0$ indicates no growth. Thus, the incremental gross potential market for the second-generation product, which is introduced at the end of period $t_{2}=\tau$, is $m_{2}=m_{1}\left((1+g)^{\tau}-1\right)$. This growth process continues throughout the planning horizon; in general $m_{i+1}=\left[(1+g)^{\tau}-1\right] \sum_{k=1}^{i} m_{k}$ for $i \geq 1$. Note that the introduction of a new generation is required to take advantage of growth in the potential market: $m_{i}$ does not change during generation $i$ 's lifetime. However, the longer the time between generations, the greater the expansion in the potential market (due to pent-up demand for higher performance, for example). 
We assume that the firm has the necessary production capacity and capabilities to serve the resulting market. Thus we ignore costs such as the costs of capacity expansion, training and inventory in our model. For example, we assume that possible decisions regarding inventory will not affect the firm's new product development decisions.

Thus in our model the optimal pace $1 / \tau^{*}$ depends on a complex set of factors: margin decay rate, sales level (which depends on the diffusion process), product development cost, and discount factor. Given the complexity and the fact that profit as a function of $\tau$ is not concave (as we show later through an example), we find $\tau^{*}$ numerically through a grid-search algorithm over all possible integer values of $\tau$, as described in Algorithm 1:

\section{Algorithm 1}

Let $\Pi(\tau)$ denote total discounted profit over all periods. Let $\tau_{\text {intro }}^{*}$ denote the optimal $\tau$ given that the firm introduces a new product, and let $\Pi\left(\tau_{\text {intro }}^{*}\right)$ denote the profit associated with $\tau_{\text {intro }}^{*}$.

1. Set $\tau=1, \tau_{\text {intro }}^{*}=0$, and $\Pi\left(\tau_{\text {intro }}^{*}\right)=-\infty$.

2. The number of generations over $T$, denoted by $M$, is $M=\lceil T / \tau\rceil$.

3. For $i=2$ to $M$, $t_{i}=(i-1) \tau ; t_{i+1}=i \tau$.

For $t=t_{i}$ to $t_{i+1}$, compute $S_{i-1}(t)$ and $S_{i}(t)$ by (4).

4. $\Pi(\tau)=\sum_{i=1}^{M} \sum_{t=0}^{T} e^{-\alpha t} S_{i}(t) r_{i}(t)-\sum_{i=2}^{M} D[1 /(\exp (d \tau)-1)+d \tau] e^{-\alpha t_{i}}$

5. if $\Pi(\tau)>\Pi\left(\tau_{\text {intro }}^{*}\right)$

$$
\begin{aligned}
& \tau_{\text {intro }}^{*}=\tau ; \Pi\left(\tau_{\text {intro }}^{*}\right)=\Pi(\tau) \\
& \tau=\tau+1 \\
& \text { if } \tau=T \text { stop; else Go to Step } 2
\end{aligned}
$$

else

$$
\tau=\tau+1
$$

End

$$
\text { if } \tau=T \text { stop; else Go to Step } 2
$$

End of Algorithm 1

The end-result of the above algorithm is $\tau_{\text {intro }}^{*}$, the optimal time between product introductions if the firm is constrained to introduce a product at least once during the planning horizon $T$. We note that $\tau_{\text {intro }}^{*}$ is not necessarily the solution to our problem, because the firm 
also has the option of not introducing another product in the market at all (that is, all net revenues are derived from sales of the first-generation product until the end of the planning horizon $T$ ). Thus, the optimal time between product introductions is

$$
\tau^{*}= \begin{cases}\tau_{\text {intro }}^{*} & \text { if } \Pi\left(\tau_{\text {intro }}^{*}\right)>\sum_{t=0}^{T} r_{1}(t) e^{-\alpha t} m_{1} F_{1}(t), \\ \infty & \text { otherwise }\end{cases}
$$

\section{Numerical Analysis}

In this section we describe an extensive numerical analysis to gain insights into the solution of (5). We ran a full-factorial experimental design for seven model parameters. Without loss of generality, we maintain the following parameters as fixed: $m_{1}=10$ and $r_{0}=1$. Further, we consider a long planning horizon $T=200$ periods. Hereafter, we will assume one period to be equal to one month, and thus the planning horizon corresponds to about sixteen years.

\subsection{Parameter Levels}

The parameters and levels examined are as shown in Table 2. All parameters are studied at three levels: low, medium and high. The levels for the discount factor are $0.5 \%$ per period ( $\alpha$ $=0.005), 1 \%$ per period, and $1.5 \%$ per period; this corresponds to annual discount factors of $5.8 \%, 11.3 \%$, and $16.5 \%$ respectively. The levels for the PD cost scaling parameter $D$ are 12.5 , 15, and 19; this corresponds to minimum product development costs of 20, 25, and 30. To understand these numbers, consider that a minimum PD cost of 30 indicates a full quarter of earnings if the sales level is kept at its maximum for the first-generation product, without cannibalization from the second-generation product. The levels for the PD cost parameter $d$ are 0.02, 0.05, and 0.08, which result in the PD cost curves previously shown in Figure 3. 
Table 2: Experimental design

\begin{tabular}{lc}
\hline Factor & Factor Levels \\
$\alpha$ & $0.005,0.01,0.015$ \\
$D$ & $12.5,15,19$ \\
$d$ & $0.02,0.05,0.08$ \\
$b$ & $0.01,0.025,0.05$ \\
$p+q$ & $0.1,0.4,0.8$ \\
$q / p$ & $1,10,50$ \\
$g$ & $0,0.005,0.01$ \\
\hline
\end{tabular}

The levels for the decay in profit margin with time are $1 \%$ per period $(b=0.01), 2.5 \%$ per period, and $5 \%$ per period (or $11.3 \%, 25.9 \%$ and $45.1 \%$ per year). To justify these values, we consider the findings in Blackburn et al. (2004), who report value decay rates to be $1 \%$ per month for power tools (over a five-year life cycle) and 4-5\% per month for personal computers (over an 18-month life cycle).

Regarding the Bass coefficients, rather than using the parameters $p$ and $q$ individually we consider the sum $p+q$ to be one parameter and the ratio $q$ / $p$ to be another, due to the way the sum and ratio define diffusion as seen by (1). The levels for $p+q$ and $q / p$ capture a variety of diffusion processes encountered in practice and are inspired by the values used in the experimental study in Krishnan et al. (1999). We leave the values fixed over the time horizon as N/B (1992) find the values of $p$ and $q$ do not change from generation to generation in an empirical study.

Finally, the levels of market growth $g$ range from a no growth scenario with $g=0$ (stable market), to $g=0.005$ per period (corresponding to $6.2 \%$ per year), to $g=0.01$ (corresponding to $12.7 \%$ per year). Negative market growth (i.e., decline) is not considered. In this case, a pacing strategy would not be appropriate. Clearly, negative growth would increase the time between a firm's introductions. However, the firm would need to assess whether it would be beneficial to incur the product development cost to introduce another generation, or just offer the current generation until the market declines to zero. 
In total, there are $3^{7}=2,187$ experimental cells. We implement Algorithm 1 in MATLAB (1996). We first illustrate the profit function using an example taken from our experimental design, followed by an analysis of results from the entire experiment.

\subsection{Example of Profit Maximization}

Consider $\alpha=0.005$ (discount factor of $5.8 \%$ per year), $D=19$ (minimum PD cost equals $30), d=0.02, b=0.01$ ( $1 \%$ profit margin decay per month), $p+q=0.4, q / p=10$, and $g=0.005$ (6.2\% potential market growth per year). We subsequently refer to this as Example 1.

Figure 4 plots $\Pi(\tau)$ for this example; note that the curve is not concave, which necessitates our grid-search algorithm for finding the optimal time between product introductions $\tau^{*}$, which is $\tau^{*}=29$ months for Example 1. Thus, the optimal pace is $1 / \tau^{*}=(1 / 29)$ $(12)=0.41$ introductions per year, and $\Pi\left(\tau^{*}\right)=1,304$. Figure 5 plots sales and undiscounted net revenues (i.e., profits without considering product development cost) as a function of time for all seven generations during the planning horizon $T$. After each generation is introduced, undiscounted net revenue per period increases due to diffusion (and consequently higher sales per period), despite the fact that the unit profit margin decreases exponentially with time after each generation's introduction; it then peaks and decreases with time afterward, until a new generation is introduced again. The gross potential market for the first generation is 10 , and increases in gross potential market for generations two through seven (over the previous generation) are $1.56,1.80,2.08,2.40,2.78$, and 3.21 , respectively. 
Figure 4: Plot of total profit $\Pi(\tau)$ for Example 1

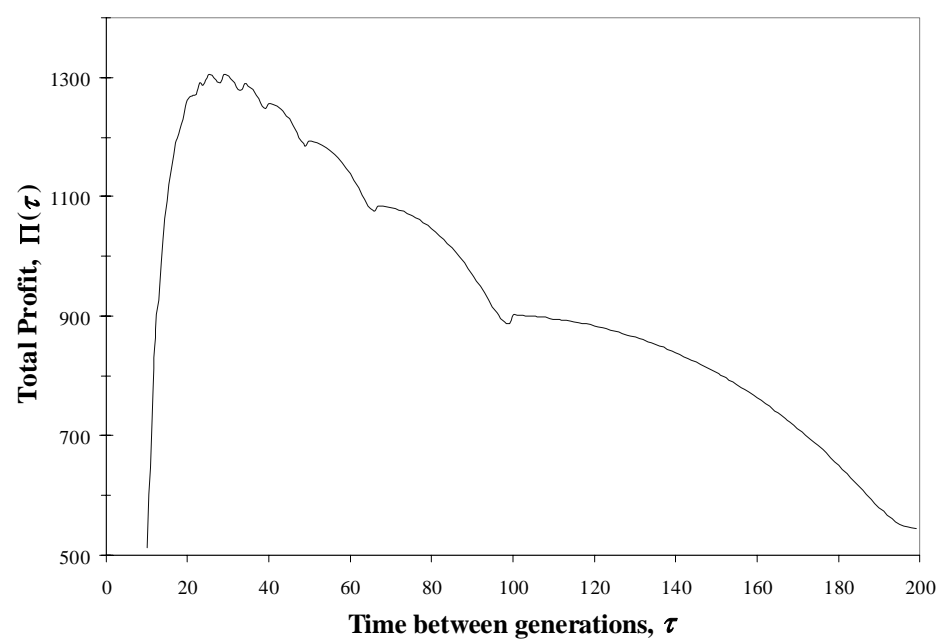

Figure 5: Sales and undiscounted net revenues per period versus time for Example 1

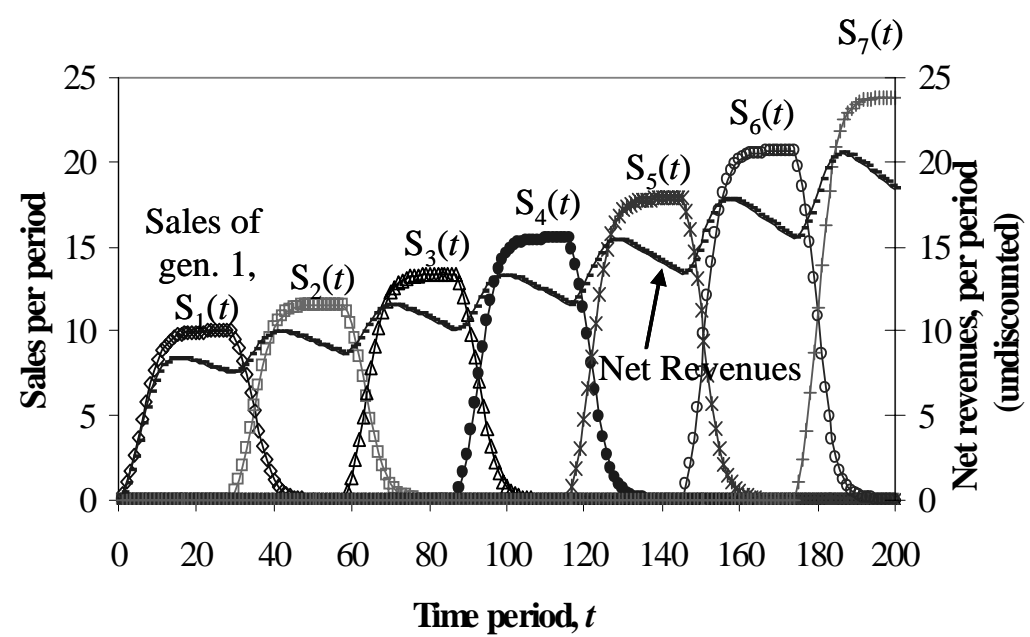

\subsection{The Main Effects of the Seven Parameters}

We now focus on the results for the entire experimental design. The statistics for the pace $1 / \tau^{*}$ are displayed in Table 3, for the 2113 cells $(96.7 \%)$ where it is optimal to introduce more than the first-generation product in the planning horizon; in 74 cells $(3.3 \%)$ it is not optimal to introduce the second-generation product. The median value of pace is 0.6 introductions/year; this number is similar to life cycles of approximately 1.75 years for some electronics products (i.e., personal computers). We note that the minimum value of pace is 0.18 
introductions/year or a life cycle of 5.6 years, which is considerably lower than the planning horizon; we believe that this result reinforces our belief that the choice of planning horizon $T$ is not critical to the insights our model generates, as long as $T$ is "large enough". The maximum pace is 1.5 introductions per year, or 8 months between introductions.

Table 3: Statistics for the optimal pace (in introductions per year)

\begin{tabular}{lc}
\hline Statistic & Value \\
\hline Minimum & 0.18 \\
$25^{\text {th }}$ percentile & 0.41 \\
Median & 0.60 \\
$75^{\text {th }}$ percentile & 0.80 \\
Maximum & 1.50 \\
\hline
\end{tabular}

We first analyze the 74 cells where it is not optimal to introduce a product. To that end, we analyze the proportion in which each factor is observed at each of its three levels in those cells. If a factor does not influence the decision to never introduce a product, then we should expect that the factor is observed at each of its three levels (low, medium, high) at roughly 33\%; this can be tested statistically. The results shown in Table 4 reveal that the factors $\alpha$ and $D$ do not strongly influence the decision to not introduce the second product, since they are observed at each of their three levels in proportions not significantly different than $1 / 3$. All other factors influence the decision to not introduce the second product, although the more significant impacts are clearly due to the diffusion curve parameters $p+q$ and $q / p$. In particular, for $100 \%$ of cases where it is optimal to not introduce a product, the parameter $p+q$ is observed at its low level, and in $84 \%$ of cases the parameter $q / p$ is observed at its high level; these are very slowdiffusion types of scenarios. In addition, in $62 \%, 77 \%$, and $66 \%$ of cases where it is optimal to not introduce a product, the parameters $d, b$, and $g$ are observed at their high, high, and low levels, respectively. In short, it is not optimal to introduce a product in the planning horizon in scenarios where products diffuse very slowly, the profit margin decay is high (such that it is more difficult for the firm to finance product development), the potential market growth rate is zero or 
very low (a relatively mature market), and the PD cost is not "flat" around its minimum (high value of $d)$.

Table 4: Results of the 74 experiments where it is not optimal to introduce the second product

\begin{tabular}{lccccccc}
\hline \multicolumn{1}{c}{ Statistic for factor } & $\alpha$ & $\boldsymbol{D}$ & $\boldsymbol{d}$ & $\boldsymbol{b}$ & $\boldsymbol{p}+\boldsymbol{q}$ & $\boldsymbol{q} / \boldsymbol{p}$ & $\boldsymbol{g}$ \\
\hline Number of occurrences at low level & 15 & 16 & 7 & 6 & 74 & 0 & 49 \\
Number of occurrences at medium level & 23 & 21 & 21 & 11 & 0 & 12 & 18 \\
Number of occurrences at high level & 36 & 37 & 46 & 57 & 0 & 62 & 7 \\
& & & & & & & \\
& 20 & 22 & $9^{*}$ & $8^{*}$ & $100^{*}$ & $0^{*}$ & $66^{*}$ \\
Percent observed at low level & 31 & 28 & 28 & $15^{*}$ & $0^{*}$ & $16^{*}$ & 24 \\
Percent observed at medium level & 49 & 50 & $62^{*}$ & $77^{*}$ & $0^{*}$ & $84^{*}$ & $9^{*}$ \\
Percent observed at high level &
\end{tabular}

* Significantly different than $33 \%$ at the $\mathrm{p}=0.001$ level.

We now consider the $96.6 \%$ of cells $(2,113$ cells $)$ where it is optimal to introduce products in the planning horizon, that is, $\tau^{*}<T$. Our main objective is to provide insights as to which factors are the most important in the decision regarding the frequency of introductions for future generations of products, or the time between product introductions. To that end, we perform a regression analysis where the dependent variable is $1 / \tau^{*}$ and the independent variables are the experimental factors; consequently there are 2,113 observations for the regression analysis. This approach is also used in Bayus (1997) and Morgan et al. (2001) to summarize first-level effects for a complex model. The regression results are displayed in Table 5.

Table 5: Regression results for the optimal pace (introductions/year) for the 2,113 cells where it is optimal to introduce products

\begin{tabular}{clrrl}
\hline Importance* $^{*}$ & Factor & Coefficient & $\boldsymbol{t}$ Stat & P-value \\
\hline NA & Intercept & 0.19 & 9.77 & $<0.0001$ \\
7 & $\alpha$ & -3.25 & -4.98 & $<0.0001$ \\
6 & $D$ & -0.01 & -11.42 & $<0.0001$ \\
2 & $d$ & 4.87 & 44.64 & $<0.0001$ \\
4 & $b$ & 4.05 & 24.80 & $<0.0001$ \\
1 & $p+q$ & 0.50 & 53.71 & $<0.0001$ \\
5 & $q / p$ & -0.002 & -15.23 & $<0.0001$ \\
3 & $g$ & 20.81 & 31.75 & $<0.0001$ \\
& & & & \\
& F-statistic & 1000.63 & & \\
& Adj-R & & & \\
\end{tabular}

* This is a ranking of the factors based on the absolute value of the t-statistic. 
We first note that all regression coefficients are significant at the $p=0.0001$ level, thus all factors influence pace. The magnitude of this influence can be assessed by comparing the absolute value of the t-statistic across factors (Wagner 1995), which is given in the fourth column of Table 5; a higher absolute value of the t-statistic implies a more influential factor. Moreover, the sign of the $\mathrm{t}$-statistic also provides the direction of the relationship-a positive sign indicates that pace increases as the factor increases. Given the levels in our experimental design, the factors that most influence pace are, in order of importance: diffusion curve shape parameter $p+q(+)$, shape factor for PD cost curve $d(+)$, potential market growth rate $g(+)$, profit margin decay rate $b(+)$, diffusion curve shape parameter $q / p(-)$, and, to a lesser extent, the scale factor for the PD cost curve $D(-)$ and the discount factor $\alpha(-)$.

We now turn to the implications of these same factors on profit, given that we have optimized profit by choosing pace. Regression results are shown in Table 6. The two most significant factors are discount rate and potential market growth rate, as one would expect. However, it is interesting that the sum of the diffusion coefficients $(p+q)$ is more significant than the margin decay rate $b$. Thus the diffusion process is very significant in predicting profits, as confirmed by the use of the Bass model for forecasting.

Table 6: Regression results for profit for the 2,113 cells where it is optimal to introduce products

\begin{tabular}{|c|c|c|c|c|}
\hline \multicolumn{2}{|c|}{ Importance* Factor } & Coefficient & $t$ Stat & $P$-value \\
\hline & Intercept & $1,187.7$ & 30.11 & $<0.0001$ \\
\hline 1 & $\alpha$ & $-65,181.5$ & -48.76 & $<0.0001$ \\
\hline 6 & $D$ & -8.0 & -3.92 & 0.0001 \\
\hline 7 & $d$ & 662.5 & 2.97 & 0.0030 \\
\hline 4 & $b$ & $-10,983.9$ & -32.91 & $<0.0001$ \\
\hline 3 & $p+q$ & 752.0 & 39.15 & $<0.0001$ \\
\hline 5 & $q / p$ & -4.6 & -17.54 & $<0.0001$ \\
\hline \multirow[t]{3}{*}{2} & $g$ & $62,260.6$ & 46.44 & $<0.0001$ \\
\hline & F-statistic & 997.5 & & \\
\hline & Adj-R ${ }^{2}$ & 0.77 & & \\
\hline
\end{tabular}

* This is a ranking of the factors based on the absolute value of the t-statistic. 
To better discuss these results, we provide a visual representation of these relationships. To that end, for each factor level (e.g. $b=0.005)$, we compute the average value of $1 / \tau^{*}$, and the average value of total discounted profit over the planning horizon $\Pi\left(\tau^{*}\right)$, across all respective experimental cells (where it is optimal to introduce). This shows the average impact of each factor level on the two performance measures of interest. The results are shown in Figure 6 for the discount factor $\alpha$ and margin decay parameter $b$, Figure 7 for the PD cost curve parameters $D$ and $d$, Figure 8 for the diffusion curve parameters $p+q$ and $q / p$, and Figure 9 for the potential market growth parameter $g$. The vertical scales for all figures are the same. As expected, all parameters (Figure 6 through Figure 9) have a strong impact on profit, except for the PD cost curve parameters $d$ and $D$ (Figure 6); this is due to the fact that PD costs represent in our experiments a relatively small fraction of the total profit over the long planning horizon.

Confirming the regression results, Figure 6 (left) shows that the discount factor $\alpha$ does not have a large impact on pace. Figure 6 (right), however, shows that the higher the margin decay parameter $b$, the faster the pace $1 / \tau^{*}$. Previous work suggests that fast clockspeed industries have shorter product life cycles, equivalently, a faster pace (Mendelson and Pillai 1999, Souza et al. 2004). We confirm that as industry clockspeed (as measured by the margin decay rate $b$ ) increases, the pace $1 / \tau^{*}$ increases. The impact of the PD cost scale parameter $D$ on pace is as expected (Figure 7 left) - as $D$ increases, pace decreases - a higher PD cost implies a longer time between product introductions. Clearly, the impact of $D$ on pace would be more significant — a steeper curve — if we had used higher values of $D$ in our experimental design. Our choice of values of $D$ is such that it allows for a wide range of $1 / \tau^{*}$ values, which improves the quality of the insights. Similarly, Figure 7 (right) shows that as $d$ increases, pace increases-a higher $d$ means that PD cost increases more sharply as pace decreases, thus offering an incentive for the firm to not delay a product introduction for long. 
The diffusion parameters $p+q$ and $q / p$ (Figure 8 ) have a very significant impact on pace-a higher value of $p+q$ (lower value of $q / p$ ) indicates a faster diffusion process, and consequently the pace is faster. This is a very significant result, because it clearly demonstrates the importance of a product's diffusion process in the timing of product introductions. To the best of our knowledge, this is the first paper to show the importance of diffusion pace in the frequency of product introductions in a multi-generational product scenario.

Finally, Figure 9 shows that pace increases with a higher growth rate $g$. A faster growing market provides incentives for firms to introduce products more frequently. We assume that a firm can capture growth in the market only at the time of a new product introduction. In other words, according to the Bass model, we know the potential market and it is fixed. When we introduce the next generation, we estimate that generation's potential market, factoring in the growth parameter $g$. Our result can be interpreted as agreeing with innovation theory; for example, Abernathy and Utterback (1978) posit that in the beginning of the industry life cycle, a period of high demand growth, the focus is on product innovation, and the pace of new products is faster than in the maturity stage of the industry life cycle, a period of much lower growth. We similarly see that in faster growth conditions, i.e., $g=6.2 \%$ per year or $12.7 \%$ per year, we predict a faster pace than a mature product with zero expected growth over time (i.e., $g=0$.) 
Figure 6: Optimal pace and profit vs. discount factor $\alpha$ and margin decay parameter $b$
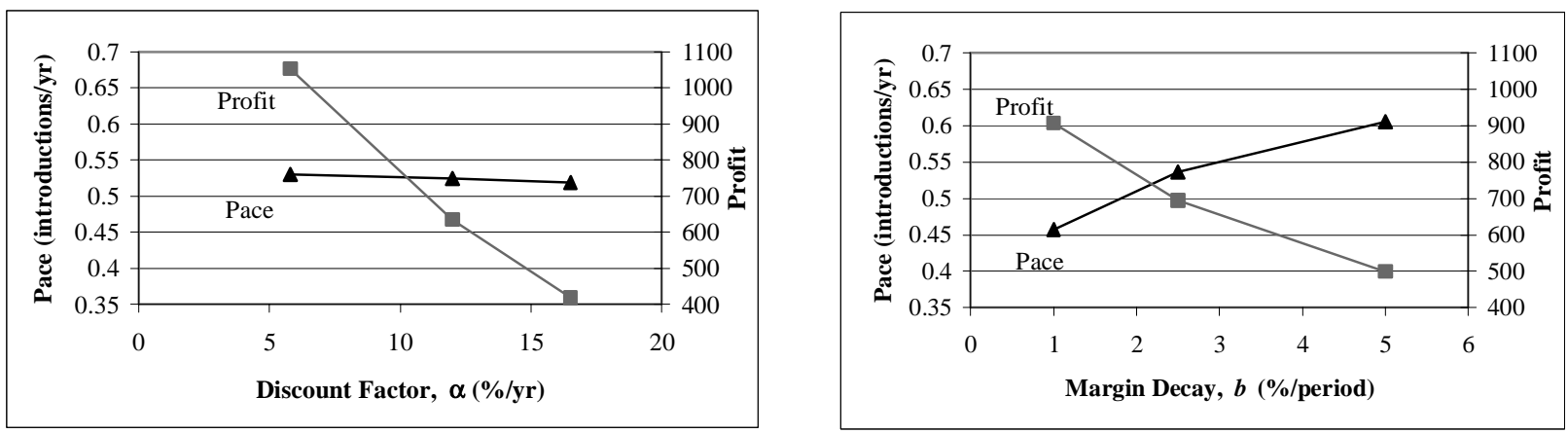

Figure 7: Optimal pace and profit vs. PD cost parameters $D$ (scale) and $d$ (shape)
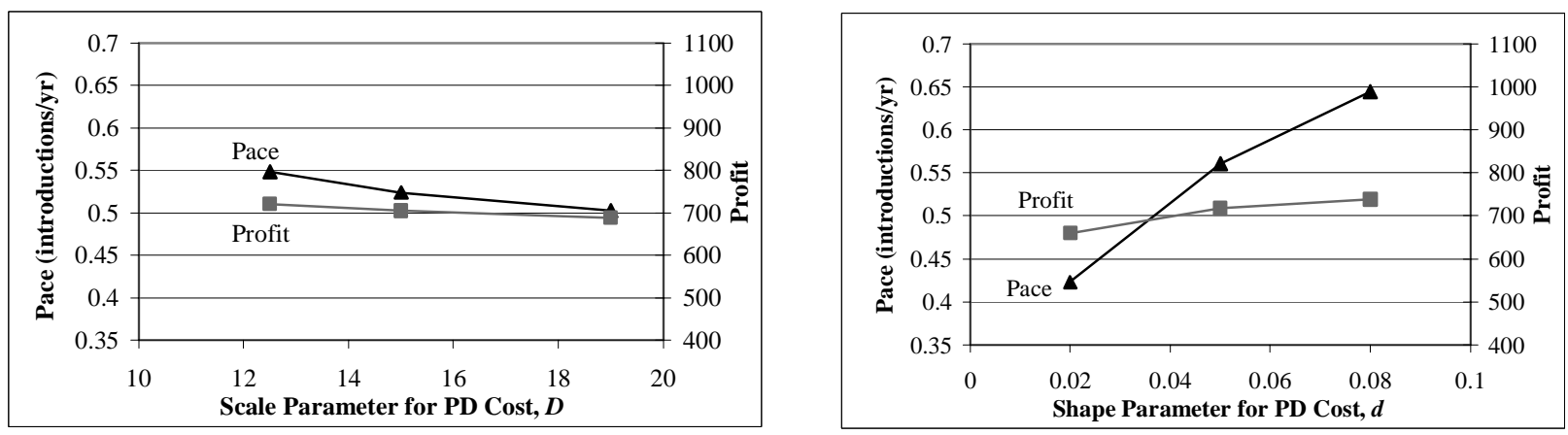

Figure 8: Optimal pace and profit vs. diffusion coefficients $p+q$ and $q / p$
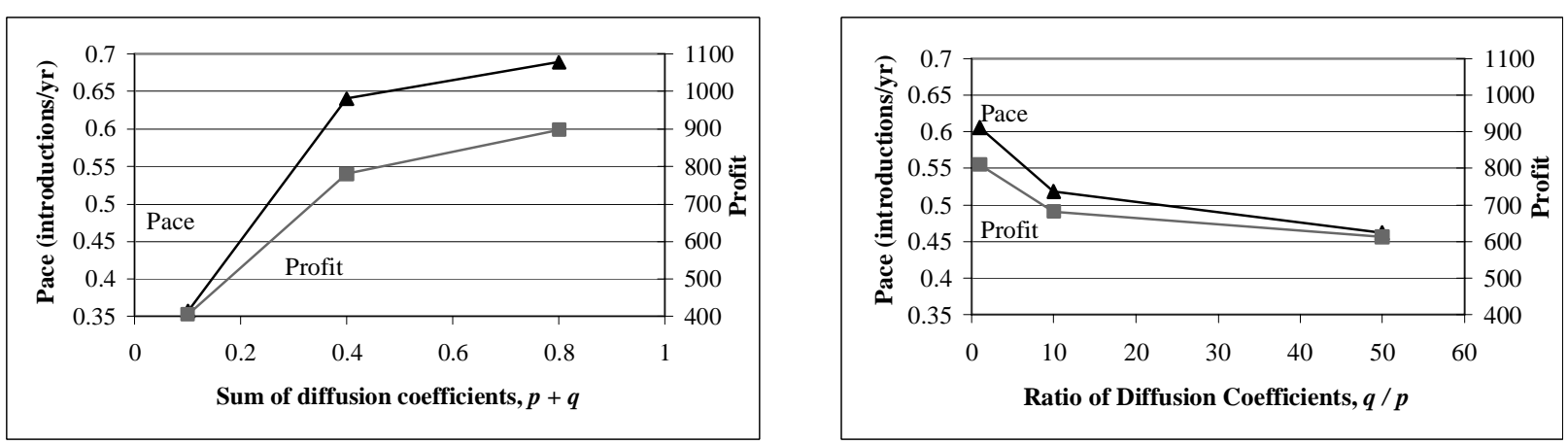
Figure 9: Optimal pace and profit vs. growth parameter $g$

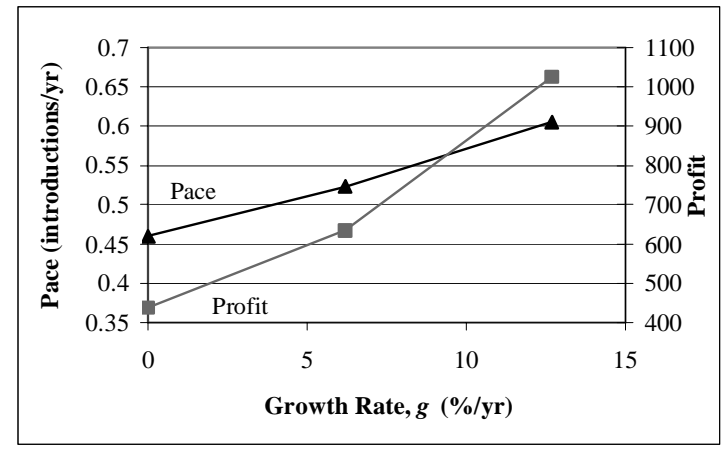

\subsection{Two-Factor Interactions}

Two-factor and higher-level interactions were also investigated using SPSS 12.0 software (SPSS 2004). For brevity, we show in Table 7 the two-factor interactions other than those involving the discount rate $\alpha$ (as this parameter was the least significant). The interactions are listed in order of their significance.

Table 7: Statistics for the two-factor interactions

\begin{tabular}{lrr}
\hline Factors & $\boldsymbol{F}$ & Level of Significance \\
\hline$p+q^{*} q / p$ & $2,027.4$ & $<0.001$ \\
$d^{*} g$ & 190.3 & $<0.001$ \\
$b^{*} g$ & 98.2 & $<0.001$ \\
$d^{*} p+q$ & 77.5 & $<0.001$ \\
$D^{*} d$ & 41.2 & $<0.001$ \\
$b^{*} q / p$ & 33.9 & $<0.001$ \\
$b^{*} p+q$ & 33.6 & $<0.001$ \\
$d^{*} b$ & 28.0 & $<0.001$ \\
$D^{*} g$ & 17.7 & $<0.001$ \\
$q / p * g$ & 15.0 & $<0.001$ \\
$p+q * g$ & 5.1 & $<0.001$ \\
$D^{*} b$ & 3.8 & 0.005 \\
$d^{*} q / p$ & 1.5 & 0.213 \\
$D^{*} p+q$ & 0.5 & 0.747 \\
$D^{*} q / p$ & 0.2 & 0.925 \\
\hline
\end{tabular}

As indicated, the most significant interaction is that between the sum and the ratio of the Bass coefficients, namely, between the factors $p+q$ and $q / p$. The nature of this interaction is illustrated in Figure 10, where we note that the horizontal axis $(q / p)$ is plotted on a log scale for 
better visualization. Note that when the sum of the coefficients is relatively high, at a level of 0.8 , the ratio of the coefficients has relatively little impact on the pace. However, if the sum is relatively low (equal to 0.1 ), then the pace drops off if the coefficient of innovation drops off (i.e., as the ratio $q / p$ increases). Speaking loosely, a fast pace may be optimal even if the customer base is dominated by imitators, but only when there is at least a sufficient base of innovators to "ignite" the diffusion process. Another informal interpretation is that innovators and imitators are substitutable, if there exists a sufficient core of innovators.

Figure 10: Interaction of the two factors $p+q$ and $q / p$

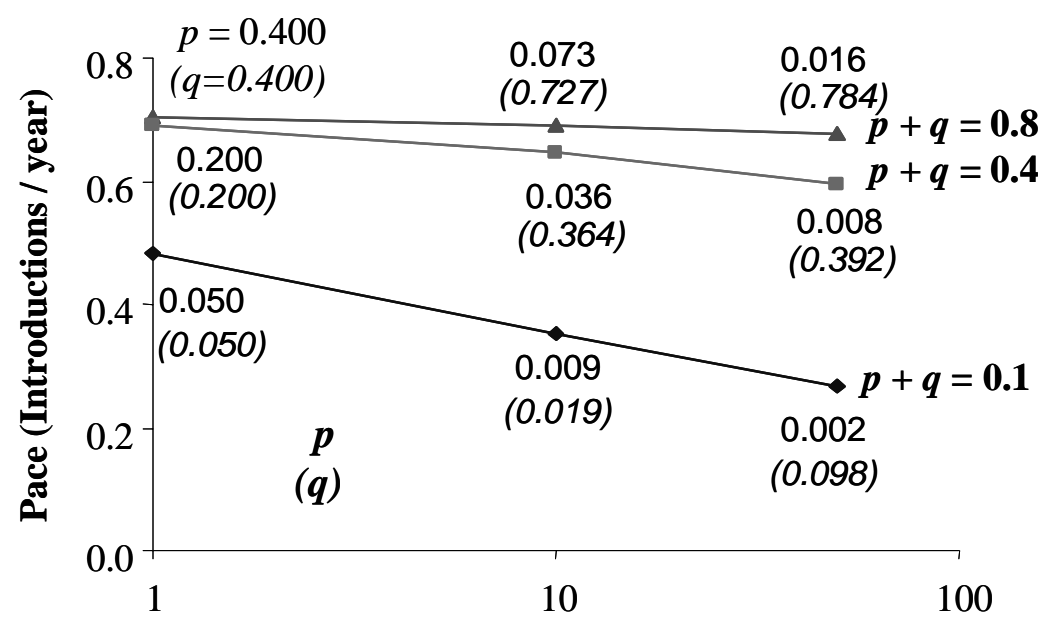

Ratio of Bass coefficients, $q$ / $p$

\section{Discussion and Summary}

We have examined how a firm might set its pace of new product introductions, as a function of a number of firm- and industry-specific factors. The intent of our model is to lend insight into some of the trade-offs that a firm faces in setting its pace of product generational updates.

The Bass (1969) and multi-generation Bass (N/B 1987, 1992) models have been successfully fitted to a variety of product types such as electronics, pharmaceuticals, and 
consumer goods. The applicability of the model and the widespread belief that many products do diffuse through the market makes this diffusion relevant to the product development decision. This is the first paper (to our knowledge) to find a significant link between the diffusion parameters and the pace of new product development.

We find that the other key drivers of pace are the shape of the product development cost curve, the market growth rate, and the rate of margin decline. The shape parameter of the new product development curve determines the minimum cost product development time. One can imagine that the nature of the development project might determine this. However, a firm's capabilities in product development would also play a role in setting this minimum and certainly in a firm's being able to successfully complete the project near the time of the minimum cost. Thus a firm's capabilities in product development are important, as a lower $d$ (i.e., a lower time at which the product development cost is minimized) allows a firm to optimally choose a faster pace. A possible implication of our results is that by modestly improving its PD cost curve, a firm achieves a significant strategic advantage. That is, driving the market at a much faster pace could have significant negative impacts on competition.

By considering different levels of potential market growth rates, we represent not only different classes of products, but also different stages in the product life cycle. We find that for more mature industries associated with slower growth rates, the optimal pace of new product introduction will be slower. For new, high growth industries, we predict the pace of new product introduction will be faster.

The inclusion of the rate of margin decline allows us to link our results to the idea of clockspeed. Faster clockspeed industries are associated with faster margin decline. Empirical studies have shown that faster clockspeed industries such as televisions and personal computers have more frequent product introductions, in other words a faster pace (Mendelson and Pillai 
1999). Our results agree with these observations. Our model leads to a firm's optimal pace. However, this pace may be different than the underlying industry pace. Previous research has shown that operating at or near industry clockspeed is optimal for maximizing profits (Souza et al. 2004). Thus if a firm's optimal pace is slower than that of the industry, it indicates a need for reexamination of the firm's internal processes that lead to factors in this model such as unit costs and product development costs.

Our model leaves open the possibility for many extensions. Our model does not explicitly consider the game-theoretic nature of the relationship between firms or between the firm and its customers. However, to the extent that these factors might implicitly be built into the rate of margin decay or the diffusion parameters, our model could be applied to settings where these factors are at play. Given our finding that the pace increases with faster margin decay, we might expect that competition hastens the margin decay and hence quickens the pace.

Similarly, it would be of interest to formalize customer considerations regarding whether to make an immediate purchase or wait for an updated model. Here we might expect that decisions to delay purchase would reduce the diffusion coefficients such that the pace would slow, but this might in turn encourage customers not to delay their purchases such that we might find an equilibrium outcome. Such a model would also include product quality as a decision variable, while here we limit ourselves to deciding only pace. We leave these extensions to future work.

\section{References}

Abernathy, W., J. Utterback. 1978. Patterns of Industrial Innovation. Technology Review 80(7), 40-47.

Bass, F. 1969. A New Product Growth Model for Consumer Durables. Management Science 15(5), 215-227. 
Bass, F. 1980. The Relationship Between Diffusion Rates, Experience Curves, and Demand Elasticities for Consumer Durable Technological Innovations. The Journal of Business 53(3) S51-S67.

Bass, F., M. Trichy, V. Krishnan, D. C. Jain. 1994. Why the Bass Model Fits Without Decision Variables. Marketing Science 13(3), 203-223.

Bayus, B. L. 1997. Speed-to-Market and New Product Performance Trade-offs. Journal of Product Innovation Management 14(6), 485-497.

Bayus, B. L., S. Jain, A. G. Rao. 1997. Too Little, Too Early: Introduction Timing and New Product Performance in the Personal Digital Assistant Industry. Journal of Marketing Research 34(1), 50-63.

Bhattacharya, S., V. Krishnan, V. Mahajan. 2003. Operationalizing Technology Improvements in Product Development Decision Making. European Journal of Operational Research 149(1), 102-130.

Blackburn, J., V. D. Guide Jr., G. C. Souza, L. Van Wassenhove. 2004. Reverse Supply Chains for Commercial Returns. California Management Review 46(2), 6-22.

Carrillo, J. 2004. Industry Clockspeed and the Pace of New Product Development. Working Paper, University of Florida.

Chen, M., C. Yu. 2002. The Optimal Launch Schedule for Two New Cannibalistic Durables: A Diffusion Theory Approach. Asia-Pacific Journal of Operational Research 19(1), 1-15.

Cohen, M., J. Eliashberg, T. Ho. 1996. New Product Development: The Performance and Timeto-Market Tradeoff. Management Science 42(2), 173-186.

Dataquest, Inc., a unit of Gartner, Inc. 2003.

Eisenhardt, K. M., S. L. Brown. 1998. Time Pacing: Competing in Markets that Won't Stand Still. Harvard Business Review 76(2), 59-69.

Fine, C. 1998. Clockspeed: Winning Industry Control in the Age of Temporary Advantage. Perseus Books, Reading, MA.

Graves, S. B. 1989. The Time-Cost Tradeoff in Research and Development: A Review. Engineering Costs and Production Economics 16(1), 1-9.

Intel, Inc. web site, http://www.intel.com/, 2004.

Klastorin, T., W. Tsai. 2004. New Product Introduction: Timing, Design and Pricing. Manufacturing and Service Operations Management 6(4), 302-320. 
Kornish, L. J. 2001. Pricing for a Durable-Goods Monopolist Under Rapid Sequential Innovation. Management Science 47(11), 1552-1561.

Krishnan, T., F. Bass, D. Jain. 1999. Optimal Pricing Strategy for New Products. Management Science 45(12), 1650-1663.

Krishnan, V., K. Ramachandran. 2004. A Product Design-Driven Approach to Managing Rapid Sequential Innovation. Working Paper, University of Texas, Austin, TX.

Krishnan, V., W. Zhu. 2003. Designing a Family of Development-Intensive Products. Working Paper, University of Texas, Austin, TX.

Mahajan, V., E. Muller, F. Bass. 1990. New Product Diffusion Models in Marketing: A Review and Directions for Research. Journal of Marketing 54(1), 1-26.

MATLAB, MATLAB Reference Guide. 1996. The MathWorks, Inc., Natick, MA.

Mendelson, H., R. R. Pillai. 1999. Industry Clockspeed: Measurement and Operational Implications. Manufacturing and Service Operations Management 1(1), 1-20.

Moorthy, K., I. Png. 1992. Market Segmentation, Cannibalization and the Timing of Product Introductions. Management Science 38(3), 345-359.

Morgan, L., R. Morgan, W. Moore. 2001. Quality and Time-to-Market Trade-offs When There are Multiple Product Generations. Manufacturing and Service Operations Management 3(2), 89104.

Norton, J., F. Bass. 1987. A Diffusion Theory Model of Adoption and Substitution for Successive Generations of High-Technology Products. Management Science 33(9), 1069-1086.

Norton, J., F. Bass. 1992. Evolution of Technological Generations: The Law of Capture. Sloan Management Review 33(2), 66-77.

Schmidt, G. M., C. T. Druehl. 2004. Diffusion of a New Product: Due to Changes in Product Attributes and Costs? Working Paper, Georgetown University.

Smith, P., D. Reinertsen. 1991. Developing Products in Half the Time. Van Nostrand Reinhold, New York.

Souza, G. C. 2004. Product Introduction Decisions in a Duopoly. European Journal of Operational Research 152(3), 745-757.

Souza, G. C., B. Bayus, H. Wagner. 2004. New-Product Strategy and Industry Clockspeed. Management Science 50(4), 537-549.

SPSS. 2004. SPSS, Inc., Chicago, IL. 
Ulrich, K. T., S. D. Eppinger. 2004. Product Design and Development. Third Edition. Irwin McGraw Hill.

Wagner, H. 1995. Global Sensitivity Analysis. Operations Research 43(6), 948-969.

Wilson, L., J. Norton. 1989. Optimal Entry Timing for a Product Line Extension. Marketing Science 8(1), 1-17.

Yelle, L. 1979. The Learning Curve: Historical Review and Comprehensive Survey. Decision Sciences 10(2), 302-328. 